\title{
A Dynamic Model for Crime Control and Policy Evaluation
}

\author{
N.U. AHMED ${ }^{\text {a** }}$ and M.A. RAHIM ${ }^{\dagger}$ \\ a Department of Electrical Engineering and Department of Mathematics, \\ University of Ottawa, 161 rue Louis-Pastuer, P.O. Box 450 Succ A, \\ Ottawa (Ontario), K1N6N5, Canada
}

(Received 5 June 1998; In final form 10 August 1998)

The paper is concerned with the development of a rigorous mathematical model describing the dynamics of criminal population subject to sentencing policies of any penal (legal) system. The model enables evaluation of the impact of preventive measures used in the society and correctional measures used by the penitentiaries. A performance index reflecting the effectiveness of such measures and the cost to the society for providing the same is introduced and the question of optimality discussed.

Keywords: Dynamic model; Crime control; Policy evaluation; Mathematical model

\section{INTRODUCTION}

Growth or decline of crime and the corresponding convicted criminal population is not a random phenomenon. It is in fact a dynamic process in the sense that there is a continuous interplay of numerous factors relating to crime and conviction such as the justice system, economic conditions, social structure and value system, psychological makeup, drug and alcohol abuse, religious beliefs, etc., which directly or indirectly cause and control the state of the process. One simple way to understand and describe the process is to use an aggregated

\footnotetext{
* Corresponding author. Tel.: 613-562-5800, ext. 6258. Fax: 613-562-5174.

E-mail: ahmed@site.uottawa.ca.

${ }^{\dagger}$ Former Statistical Advisor, Ministry of the Solicitor General and Statistics Canada, Government of Canada.
} 
model in terms of the totality of criminal incidents over a given period of time and the convicted criminal population at any given point of time. To further simplify matters and also for the immediate purpose of correctional policy making and cost control, sociologists and criminologists usually narrow down the problem to a subset of the criminal population, namely, those who have been convicted and sentenced to imprisonment.

Numerous works exist in the literature dealing particularly with the problem of forecasting the size of this subset, that is, prison population at a future point of time. This is essential for the policy makers who have to deal with the administrative costs and security issues relating to prison capacity and overcrowding. Obviously any such forecasting effort would have to depend on some kind of mathematical modeling. Most often the models are constructed based on existing theories of regression analysis, time series analysis and input-output analysis with or without some probabilistic components. Some notable works in this area are those of Stollmack [1], Blumstein et al. [2], Barnett [3], Schmidt and Witte [4,5], Visheret et al. [6] and Lattimore and Baker [7]. Stollmack's model, one of the earliest, is of the form

$$
P_{t}=P_{t-1} \mathrm{e}^{-\mu}+(\gamma / \mu)\left(1-\mathrm{e}^{-\mu}\right),
$$

where the first term on the right hand side represents the number of prisoners at time $(t-1)$ who remain in prison at time $t$, and the second term represents the number of individuals sentenced to imprisonment during the time interval $(t-1, t]$. This model requires two basic assumptions. First, the intake or sentencing rate denoted by $\gamma$ remains constant over time. Second, the negative exponential service distribution with service rate (rate of discharge) $\mu$ also remains constant over time.

Blumstein et al. [2] improved this model by applying it separately on each subset of the general population determined by the levels of each of the categories of age $(a)$, sex $(s)$, race $(r)$, and offense-specific imprisonment $(o)$ and then summing over all these subsets. His model is therefore of the form

$$
P_{t}=\sum_{a} \sum_{r} \sum_{s} \sum_{o}\left\{P_{t-1, \text { arso }} \mathrm{e}^{\mu_{a r s o}}+C_{t, \text { arso }} S_{a r s o}\left(1-\mathrm{e}^{\mu_{a r s o}}\right)\right\},
$$


where $C_{\text {arso }} \equiv \gamma_{\text {arso }} \equiv$ admission rate for the group classified as arso and $S_{\text {arso }} \equiv\left(1 / \mu_{\text {arso }}\right) \equiv$ mean time served in prison by the group identified by arso.

Barnett's model [3] was based on a number of probabilistic assumptions, such as, all prison admissions come from a chronic offender population each member of which commits crime at age $c$ and retires at age $r$. He assumes age-specific density of such offenders and assigns certain probabilities of their return to prison at time $t$.

The concept and measurement of recidivism was rigorously formulated earlier by Rahim in $[9,10]$. Schmidt and Witte $[4,5]$ incorporated the process of recidivism in their model and used standard methods of survival analysis for estimating the number of imprisonments due to repeat crime. In other words they used separate models for the "first time offenders" and those who were imprisoned for repeating crime following release.

Lattimore and Baker [7] proposed an improved model which is essentially an input-output analysis with a probabilistic component which is based on the earlier works of Stollmack, Blumstein et al. and Barnett.

These well known and pioneering works, including all other notable works in the area, do have two significant limitations. First, none of these models have taken into account the ordering of offense types in terms of their severities. Clearly the model parameters and all the inherent assumptions would have to differ from one offense category to another. For example, assumptions that would appear to be justifiable for bicycle theft would not necessarily be so for homicide or rape. Second, none of these modeling efforts have considered the suitability or applicability of what is known as "dynamic process modeling" well known in the field of Systems and Control theory which has found widespread applications in applied sciences, engineering, econometrics and management. Such models take into account causative and controlling factors which are most appropriate for not only prison population forecasting but also for evaluation of effectiveness of correctional policies in place and their possible modifications for improved results in terms of crime reduction. In other words, the most important feature is that, it enables controlling the process through a set of control parameters that is essential for objective policy making. 
The primary purpose of this paper is, therefore, to develop a mathematical framework of modeling a dynamic process for prison population forecasting and providing a tool for policy development and crime control. According to our survey of literature in the area of criminology and sociology we did not find any dynamic model as presented here. The rest of the paper is thus organized as follows. In Section 2, we develop the model; in Section 3, we discuss the questions relating to model identification and model validation; in Section 4 we spell out clearly the advantages of having a dynamic model. In Section 5, we show how policy optimization is possible through a set of preventive and correctional measures. In the conclusion section, we reiterate the usefulness of dynamic models.

\section{DYNAMIC MODELS}

In this section we develop a mathematical model for the dynamics of criminal population subject to various regulatory policies used by penitentiaries and the parole system. For this purpose, we categorize or classify the whole spectrum (column) of crime by intensity or severity. Let $\left\{C_{1}, C_{2}, C_{3}, \ldots, C_{n}\right\}$ denote the types of crime arranged in the descending order $\left\{C_{1}>C_{2}>C_{3}>\cdots>C_{n}\right\}$ of severity. Accordingly each criminal is assigned a class depending on the severity of the crime committed. Thus the entire prison population of a country, or any region of a country, or a system of penitentiaries managed by any Government agency, is partitioned into these classes. By prison population here, we mean the population that is serving their sentence while in the prison or out on parole. Clearly the population count in any of these classes varies with time. Let $\left\{x_{1}(t), x_{2}(t), x_{3}(t), \ldots, x_{n}(t)\right\}$ and $\left\{y_{1}(t), y_{2}(t), y_{3}(t), \ldots, y_{n}(t)\right\}$ denote the counts of population in the prison and on parole, respectively, in the crime categories $\left\{C_{1}, C_{2}\right.$, $\left.C_{3}, \ldots, C_{n}\right\}$ at any arbitrary time $t$. We are interested in the underlying dynamics that governs the temporal variation of the vectors

$$
x(t) \equiv\left(x_{1}(t), x_{2}(t), x_{3}(t), \ldots, x_{n}(t)\right)^{\prime} \equiv\left(\begin{array}{c}
x_{1}(t) \\
x_{2}(t) \\
\vdots \\
x_{n}(t)
\end{array}\right)
$$


and

$$
y(t) \equiv\left(y_{1}(t), y_{2}(t), y_{3}(t), \ldots, y_{n}(t)\right)^{\prime} \equiv\left(\begin{array}{c}
y_{1}(t) \\
y_{2}(t) \\
\vdots \\
y_{n}(t)
\end{array}\right)
$$

We shall call this pair of vectors the state variable and its value at time $t$ given by $z(t) \equiv\{x(t), y(t)\}$ the state at time $t$. Clearly the total population serving prison sentences at any given time is given by

$$
Z(t) \equiv X(t)+Y(t)
$$

where

$$
X(t) \equiv \sum_{i=1}^{n} x_{i}(t) \quad \text { and } \quad Y(t) \equiv \sum_{i=1}^{n} y_{i}(t)
$$

Let $P(t)$ denote the total population of the country or the region under study. Clearly $Z(t) / P(t) \in[0,1]$ and if it is larger for one country compared to another, it will be a matter of concern for that country. Similarly within the same penal system if $\left(x_{1}(t)+y_{1}(t)\right) / Z(t)$ or $\left(x_{1}(t)+\right.$ $\left.y_{1}(t)\right) / P(t)$ is larger compared to $\left(x_{i}(t)+y_{i}(t)\right) / Z(t)$ or $\left(x_{i}(t)+y_{i}(t)\right) / P(t)$ for any class $C_{i}, i \neq 1$, it is again a matter of concern to the penal system. In short these vectors of population and their variation with time are indicative of the state of security of the general population of the country or the region. Hence it appears that a dynamic model for the vector processes $\{x(t), t \in I\}$, and $\{y(t), t \in I\}$, where $I$ is an index set to be defined shortly, if available to the penal system, will be useful for evaluation of effectiveness of existing regulatory policies and even formulating alternative ones to achieve better results. We shall return to the question of regulation in the sequel. After having made the case for a dynamic model we will now develop its mathematical structure.

For the index set $I$ one can take the set of all positive and negative integers

$$
I=\{k=0 ;+1,-1 ;+2,-2 ;+3,-3 ; \ldots\} .
$$

Each one represents a point on the time horizon and the unit interval between them represents a period of one day or one week or one 
month or any other suitable length of time. Associated with each level of crime $C_{i}, i=1,2, \ldots, n$, the length of sentence may be different and it is denoted by $L_{i}, i=1,2,3, \ldots, n$, where $L_{i}=N_{i} \times$ the length of the basic time interval used for the index set $I$. The number $N_{i}$ is an integer denoting the number of basic units of time. An inmate in the class $C_{i}$ may be granted parole after serving one, two or any number of basic intervals of time but certainly not more than $N_{i}-1$. The inmate is released after completion of sentence whether or not he has been on parole. First note the basic contributors to the change of population in each class say, $C_{i}$, during any time period $[t, t+1], t \in I$. They are listed as follows:

(1) fresh convictions during the period from the rest of population,

(2) number of inmates released on parole during the period or released on completion of sentence,

(3) number of inmates returning to prison during this period for parole violation,

(4) number of inmates that exit from the system due to death or any other cause.

Corresponding to these contributors, we introduce the following fundamental coefficients:

$$
\left\{\alpha_{i}, a_{i}^{r}, b_{i, j}^{r}, e_{i}^{1}, e_{i}^{2}\right\}
$$

where

(i) $\alpha_{i}$ denotes the (fractional) growth rate of fresh recruits from the rest of the population;

(ii) $a_{i}^{r}$ denotes the fractional rate of paroles granted to inmates of class $C_{i}$ after they have served their sentence $r$ units of parole time where $r \in\left\{1,2,3, \ldots, N_{i}\right\}$. The last coefficient, $a_{i}^{N_{i}}$, denotes the fractional rate of release on completion of sentence. Note that it denotes the fraction of population, $x_{i}\left(t-N_{i}\right)$, that existed at time $\left(t-N_{i}\right)$, who have completed their full $N_{i}$ units of sentence at time $t$ and hence are released;

(iii) $b_{i, j}^{r}$ denotes the rate (a fraction) of parole violations after serving $r$ units of parole time in the class $C_{j}, j \geq i$, and then committing a crime classified as $C_{i}$. Clearly $b_{i, i}^{r}$ is the rate of relapse within the class $C_{i}$ after serving $r$ units of parole time; 
(iv) $\mathrm{e}_{i}^{1}, \mathrm{e}_{i}^{2}$ denote the exit rates of inmates of class $C_{i}$ while in the prison and while on parole respectively.

Using these coefficients (parameters) we are now in a position to write the population level at time $t+1$, dependent on the history up to time $t$, as follows:

$$
\begin{gathered}
x_{i}(t+1)=x_{i}(t)+\alpha_{i}\left(P(t)-\sum_{k=1}^{n}\left(x_{k}(t)+y_{k}(t)\right)\right)-\sum_{r=1}^{N_{i}} a_{i}^{r} x_{i}(t-r) \\
+\sum_{j=i}^{n} \sum_{r=1}^{N_{j}} b_{i, j}^{r} y_{j}(t-r)-\mathrm{e}_{i}^{1} x_{i}(t), \\
\quad i=1,2, \ldots, n, \text { and } t \geq 0 \\
y_{i}(t+1)=y_{i}(t)+\sum_{i=1}^{N_{i}-1} a_{i}^{r} x_{i}(t-r)-\sum_{j=i}^{n} \sum_{r=1}^{N_{j}} b_{i, j}^{r} y_{j}(t-r)-\mathrm{e}_{i}^{2} y_{i}(t), \\
i=1,2, \ldots, n, \text { and } t \geq 0 .
\end{gathered}
$$

Note that (2.7) and (2.8) constitute a system of $2 n$ simultaneous delay difference equations. The first term on the right hand side of Eq. (2.7) represents the prison population in class $C_{i}$ at time $t$, the second term represents the fresh recruits or convictions from the remaining population during the period $[t, t+1]$, the third term represents the number that was granted parole and release. The sum is shown to start from $r=1$; however, it is more appropriate to choose a lower limit, say, $m_{i}<N_{i}$, that corresponds to mandatory sentence of at least $m_{i}$ periods of basic time units for eligibility of parole. However, this modification is not absolutely essential since one can take $a_{i}^{r}=0$, for $1 \leq r \leq m_{i}$, and this will serve the purpose. This is more flexible since, if necessary, the parole board may choose different values for different individuals in the same category. The fourth term represents the number of relapses from the classes $\left\{C_{i}, C_{i+1}, \ldots, C_{n}\right\}$ to the class $C_{i}$. The sum starts at $i$ since it is reasonable to assume that only the inmates paroled from (lower) classes $C_{j}, i \leq j \leq n$, who have, during the parole time, committed crime of class $C_{i}$ will be admitted into the class $C_{i}$ and not those who have committed crime of greater severity. Note that if relapses occur only within the same class or, in other words, there are no crossings of categories then $b_{i, j}^{r}=0$ for all $j \neq i$. In that 
case the double sum reduces to a single sum. The last term represents the total number of exits during the period $[t, t+1]$ with $\mathrm{e}_{i}^{1}$ denoting the fractional exit rate. Similarly in Eq. (2.8) the first term on the right hand side represents the parole population at time $t$, the second term denotes the number of paroles granted during the interval $[t, t+1]$ which is negative of the third term of Eq. (2.7) with the upper limit $N_{i}$ replaced by $\left(N_{i}-1\right)$, the third term represents the number of parole violations and it is the negative of the fourth term of Eq. (2.7). The fourth term denotes the total number of exits from the parole rolls during the period $[t, t+1]$. The upper limit of the sum in the third term of Eq. (2.7) is $N_{i}$ while the second term of Eq. (2.8) has the upper limit $\left(N_{i}-1\right)$. This is due to the fact that the last component of the sum represents release on termination of sentence. That is, $a_{i}^{N_{i}}$ denotes the rate of release due to termination of sentence.

This is a system of $2 n$ delay-difference equations describing the dynamics of prison population, both in the prison and on parole. In order to solve such a system of equations it is necessary to provide the past history of population. By past history here, we mean simply the population count during the periods $\left\{-N_{i} \leq t \leq 0, i=1,2, \ldots, n\right\}$. This is described as follows:

$$
\begin{array}{ll}
x_{1}(t)=\phi_{1}(t), & -N_{1} \leq t \leq 0, \\
x_{2}(t)=\phi_{2}(t), & -N_{2} \leq t \leq 0, \\
\vdots & \\
x_{n}(t)=\phi_{n}(t), & -N_{n} \leq t \leq 0
\end{array}
$$

for the vector $x(t)$ and

$$
\begin{array}{ll}
y_{1}(t)=\psi_{1}(t), & -N_{1} \leq t \leq 0, \\
y_{2}(t)=\psi_{2}(t), & -N_{2} \leq t \leq 0, \\
\vdots & \\
y_{n}(t)=\psi_{n}(t), & -N_{n} \leq t \leq 0
\end{array}
$$

for the vector $y(t)$.

Here $\left\{\phi_{i}(t), \psi_{i}(t) ; t \in\left[-N_{i}, 0\right], i=1,2,3, \ldots, n\right\}$ denotes the past history which is known. Given the past history, Eqs. (2.7)and (2.8) determine uniquely the population vector $\{x(t), y(t), t \geq 0\}$. 


\subsection{Fundamental Parameters}

The parameters indicated in (2.6) are fundamental and they must be determined by experiment and observation over a sufficient period of time. These parameters are dependent on many factors which we wish to discuss here.

The parameters $\left(\alpha_{i}, i=1,2, \ldots, n\right\}$ depend on several factors such as (1) level of social education, (2) level of social iniquities, (3) poverty or income level, (4) family relations (background, divorce, separation), (5) lack of appropriate and adequate social support system, (6) unjust government policies, (7) fairness or lack of it in the legal system, etc. Clearly these and many others are sources of frustration and hate leading to criminal activities. According to the principle of least action, which plays a central role in classical mechanics, any physical system including biological entities attain dynamic or static equilibrium by following the path of least action or discomfort. Existence of iniquities and lack of fairness in any social system are clearly potential sources of discomfort and discord and any human being subjected to such a system will naturally attempt to minimize suffering by violating the very rules and regulations that he or she believes are the causes of suffering. In any case there are many possible remedies that a national government can choose to apply in order to improve the situation. The set of possible remedial actions or preventive measures, say $\ell$ in number, that a government can adopt may be considered as the control variables denoted by the vector

$$
u \equiv\left\{u_{1}, u_{2}, \ldots, u_{\ell}\right\}^{\prime}
$$

where the value of $u_{k}$ determines the level of effort in applying the $k$ th measure. These variables may be interpreted as the level of effort given to each individual social support system. Appropriate choice of these variables can lead to increase or decrease of the values of $\left\{\alpha_{i}\right.$, $i=1,2, \ldots, n\}$. In other words these basic coefficients $\left\{\alpha_{i}\right\}$ are functions of control measures and they are expressed by writing

$$
\alpha_{i} \equiv \alpha_{i}\left(u_{1}, u_{2}, \ldots, u_{\ell}\right), \quad i=1,2,3, \ldots, n .
$$

Since small efforts produce small results, these are continuous functions of their arguments. The parameters $\left\{a_{i}^{r}\right\}$, denoting the rate of 
paroles granted to the inmates in class $C_{i}$ after serving $r$ units of prison time, are variables that the parole system can choose in order to achieve better results. However, given the history of the subject, decision of the parole board depends on the correctional measures available in the (correctional) institutions where the inmates are placed. Similarly the parameters $\left\{b_{i, j}^{r}\right\}$ denoting the rate of relapses or coefficient of cross recidivism, are again dependent on the available correctional measures. If the objective is rehabilitation, one must consider all possible correctional measures that can be effectively used to modify criminal behavior including the causes and these tools must be made available to the correctional institutions. To name a few one may consider (1) appropriate educational programs (vocational or others) that offer promise for immediate employment upon termination of sentence, (2) psychological and physical programs designed for behavior modification and improvement of work habits, (3) medical and psychiatric programs designed to correct brain disorders, (4) social and educational programs designed to develop awareness of obligations and rights, etc. Assuming that there are $m$ such correctional measures denoted by the vector $v \equiv\left\{v_{1}, v_{2}, \ldots, v_{m}\right\}^{\prime}$, which are in force, the parameters $\left\{a_{i}^{r}, b_{i, j}^{r}\right\}$ can be written as functions of these measures:

$$
\begin{aligned}
a_{i}^{r} & =a_{i}^{r}\left(v_{1}, v_{2}, \ldots, v_{m}\right) \equiv a_{i}^{r}(v), \\
b_{i, j}^{r} & =b_{i, j}^{r}\left(v_{1}, v_{2}, \ldots, v_{m}\right) \equiv b_{i, j}^{r}(v) .
\end{aligned}
$$

Again by the same principle, small efforts producing small results, these are continuous functions of their arguments. Thus we have two sets of controls or tools:

$$
u=\left(u_{1}, u_{2}, \ldots, u_{\ell}\right)^{\prime}, \quad v=\left(v_{1}, v_{2}, \ldots, v_{m}\right)^{\prime},
$$

the first set denoting the preventive measures and the second denoting the correctional measures. Note that the parameters $\left\{\alpha_{i}, b_{i, j}^{r}\right\}$ are nonnegative and nonincreasing functions of the control measures while the parameters $\left\{a_{i}^{r}\right\}$ are nonnegative nondecreasing functions of their arguments. The larger the control efforts are the smaller are the coefficients $\left\{\alpha_{i}, b_{i, j}^{r}\right\}$ and the larger are the coefficients $\left\{a_{i}^{r}\right\}$. 


\section{MODEL IDENTIFICATION AND VALIDATION}

The process $z(t) \equiv\left(x_{1}(t), x_{2}(t), \ldots, x_{n}(t), y_{1}(t), y_{2}(t), \ldots, y_{n}(t)\right)^{\prime}$ given by the solution of Eqs. (2.7) and (2.8) subject to the initial data (2.9) and (2.10) is clearly dependent on all the parameters $\left\{\alpha_{i}, a_{i}^{r}, b_{i, j}^{r}, N_{i}, \mathrm{e}_{i}^{1}, \mathrm{e}_{i}^{2}\right\}$. Denoting by $p$ the assemblage of all these parameters, we can write the system of Eqs. (2.7), (2.8) and (2.9), (2.10) in the vector form

$$
\begin{aligned}
z(t+1) & =F\left(p, z(t), H_{t} z\right), \quad t \in[0, T], \\
H_{0} z & =\Phi \equiv\{\phi, \psi\}^{\prime}
\end{aligned}
$$

where $H_{t} z$ denotes the history of $z$ up to time $t$ given by

$$
H_{t} z \equiv z(t+\theta) \equiv\left\{z_{i}(t+\theta)\right\}^{\prime}, \quad \theta \in\left[-N_{i}, 0\right], \quad i=1,2,3, \ldots, n,
$$

and $F$ denotes the vector function representing the expression on the right hand side of the system of Eqs. (2.7) and (2.8) and $\phi$ denotes the vector of initial history given by Eqs. (2.9) and (2.10). System (2.7)-(2.10) is written in the abstract form (3.1) just for discussion on conceptual matters relating to modeling and validation of models. Examining the system (3.1) it is evident that the temporal evolution of the state $z$ depends on the history $\Phi$ and the parameter vector $p$ and hence the regulatory policies $\{u, v\}$ used. In particular, the state $z(t)$ at time $t$ depends on the policies $\{u, v\}$ up to time $t$. For a given $\Phi$, the state $z$ is a function of time and $p$ denoted by $z(t, p)$. If the historical record is available for a period, say, $\left[0, T_{0}\right]$ it is possible to determine the vector $p$ by minimizing the discrepancy between the model response $z(t, p)$ corresponding to any $p$, and the observed historical data $z_{0}(t), t \in\left[0, T_{0}\right]$. A simple and popular measure of discrepancy is given by the sum of the squares of Euclidean distances at each point of time between the model response and the observed data:

$$
E(p) \equiv \sum_{t \in\left[0, T_{0}\right]}\left\|z(t, p)-z_{0}(t)\right\|^{2} .
$$

The parameter vector $p^{*}$ that minimizes this error functional is called optimal and the criterion is known as the least-squares criterion. The optimal parameter $p^{*}$ can be determined by using the optimization 
algorithm given in [11, Algorithm C, p. 307]. Note that $p^{*}$ is dependent on the length of observation $T_{0}$. Thus $p^{*}$ can be updated as new observable data become available with the passage of time. It is expected that after sufficient observation $p^{*}$ will settle down at a fixed point. The vector $p^{*}$ so obtained can be considered as the true parameter. Replacing $p$ by $p^{*}$ in Eq. (3.1) we have a dynamic model for a system which is characterized by the sentencing policies and the set of preventive and correctional measures it employs. It is important to note that any change in these measures will result in a corresponding change in $p^{*}$. But $p^{*}$ can be easily adapted to any such changes by following the optimization procedure as outlined above.

The functional dependence of $p^{*}$ or equivalently the parameters $\left\{\alpha_{i}, a_{i}^{r}, b_{i, j}^{r}, N_{i}, \mathrm{e}_{i}^{1}, \mathrm{e}_{i}^{2}\right\}^{*}$ on the control measures $\{u, v\}$ is apparently a subject of great interest and challenge to social and behavioral scientists. However from a practical point of view it is possible to develop empirical relations based on records of data on Cause and Effect by following the procedure discussed above.

Often in the literature of criminology, a measure of effectiveness of the penal system is described by a factor called recidivism. One can introduce various measures of recidivism once the fundamental parameters $\left\{b_{i, j}^{r}\right\}$ have been identified through the procedure as described above. For example, the quantity $\beta_{i j}, i \leq j \leq n$, given by

$$
\beta_{i j} \equiv\left(1 /\left(N_{j}-1\right)\right) \sum_{r=1}^{\left(N_{j}-1\right)} b_{i j}^{r}
$$

is a measure of average cross recidivism from class $j$ to class $i$. This means that members on parole from a less severe crime category $C_{j}$ recommitted crime of a higher severe category $C_{i}, i \leq j$, at an average rate of $\beta_{i, j}$. Note that this is a (triangular) matrix of recidivism with all the entries below the diagonal being zero. From this we can also introduce the recidivism, $\delta_{i}$, associated with the class $C_{i}$, by summing the elements of the $i$ th row,

$$
\delta_{i} \equiv \sum_{j=1}^{n} \beta_{i j}, \quad i=1,2,3, \ldots, n .
$$

Thus by constructing a detailed dynamic model we are not only able to forecast the future prison population, but also provide the full 
spectrum of criminal population by crime type and gain knowledge of fundamental parameters such as recidivism as discussed above.

Comment Due to presence of uncertainties in population dynamics, it is natural to consider appropriate extensions of our model (3.1) to its stochastic counter part. This will be considered in our future work.

\section{ADVANTAGES OF DYNAMIC MODELS}

The advantages of a dynamic model are manifold. We explore here some of these advantages. Once a dynamic model has been set up using the procedure outlined in the preceding section, the model can be used for several purposes.

(1) Projection of prison population in the future years which is very useful for the management of penitentiary capacity. Denoting by $\tau$ the present time, this is simply done by solving the system of Eqs. (2.7) and (2.8) using the current population $z(\tau)$, and the past history $H_{\tau} z$ as defined by the expression following Eq. (3.1).

(2) Evaluation of cross and self-recidivism $\beta_{i, j}, j \leq n$, or simply the class recidivism $\left\{\delta_{i}, i=1,2, \ldots, n\right\}$. These numbers give the frequency of repeat crimes associated with each class of crime. For example, if the class $C_{1}$ denotes homicide, and if the associated $\delta_{1}$ is comparable or even larger than $\left\{\delta_{i}, i \neq 1\right\}$, there is a cause of great concern. This situation may demand reassessment of existing sentencing, social and correctional policies.

(3) System parameters can be adapted to changes in social policies and correctional measures and hence to different crime and justice systems. Computer simulation results can be used as reliable guidelines for decision making.

(4) System model can be used for the purpose of determining the optimal preventive and correctional measures required to achieve a given social objective. This is further discussed in Section 5 .

\section{OPTIMAL POLICY}

Given the model (2.7)-(2.8) with the initial history (2.9)-(2.10), there are several possible applications as indicated above, one of them being 
optimization using appropriate preventive and correctional measures. This requires an objective functional which reflects the goal of the society. For example, if the goal of the society is to reduce crime and the criminal population of the country within a specified period of time, say, $[0, T]$ starting from time zero, then one could choose the functional

$$
J(u, v) \equiv \sum_{i=1}^{n} w_{i}\left(x_{i}(T)+y_{i}(T)\right)
$$

as the objective functional, where $\left\{w_{i}, i=1,2, \ldots, n\right\}$ are the weights $\left(w_{i} \geq 0\right)$ or emphasis given to crime types. One may decide to give the largest weight to the topmost crime level and the least weight to the lowest crime level. Note that the solution vector $z(t) \equiv\{x(t), y(t)\}$, $t \in[0, T]$, a $2 n$ vector valued function, depends on the choice of control policies. In general the control measures must be nonnegative and, further, there are resource limitations, given that there are other priorities. Thus the control policies consisting of preventive and correctional measures are constrained as follows:

$$
\begin{aligned}
& U \equiv\left\{u \in R^{\ell}: 0 \leq u_{k} \leq \gamma_{k}, k=1,2, \ldots, \ell\right\} \\
& V \equiv\left\{v \in R^{m}: 0 \leq v_{k} \leq \delta_{k}, k=1,2, \ldots, m\right\}
\end{aligned}
$$

where $\left\{\gamma^{\prime} \mathbf{s}, \delta^{\prime} \mathbf{s}\right\}$ are the upper limits of available resources. Hence the problem is to find a control policy $\{u(t), v(t), t \in[0, T]\}$ subject to the constraints (5.2) that minimizes the functional

$$
J(u, v)=\sum_{i=1}^{n} w_{i}\left(x_{i}(T)+y_{i}(T)\right) \equiv \sum_{i=1}^{n} w_{i}\left(x_{i}(T, u, v)+y_{i}(T, u, v)\right) .
$$

To emphasize the dependence of the solution vector on the control policies adopted, we have written $x(T)=x(T, u, v), y(T)=y(T, u, v)$.

For maintenance of correctional institutions and parole system, there is a cost involved. If one wishes to include this cost, the objective functional (5.1) is replaced by

$$
J(u, v)=\sum_{i=1}^{n} w_{i}\left(x_{i}(T)+y_{i}(T)\right)+\sum_{t \in[0, T]}\left(\sum_{i=1}^{\ell} \lambda_{i} u_{i}(t)+\sum_{i=1}^{m} \nu_{i} v_{i}(t)\right),
$$


where $\lambda_{i}, \nu_{i}$ denote the cost per unit time, of administering the control measures.

Using modern control theory and computer programs based on this theory [11] one can determine the optimum policies. Certainly these policies can be used as a guideline by the penal system to improve the performance. Before concluding this section we wish to note that a far more general criterion than that represented by the expression (5.3) is given by

$$
J(u, v) \equiv \sum_{t \in[0, T]} \Psi(x(T, u, v), y(T, u, v), u(t), v(t))
$$

where $\Psi$ is any continuous nonnegative function of its arguments. In fact there is no difficulty in optimizing this functional (see [11]). If it is necessary to assign different weights for those in the prison and those on parole, the objective functional (5.3) can be modified as follows:

$$
J(u, v) \equiv \sum_{i=1}^{2 n} w_{i} z_{i}(T)+\sum_{t \in[0, T]}\left(\sum_{i=1}^{\ell} \lambda_{i} u_{i}(t)+\sum_{i=1}^{m} \nu_{i} v_{i}(t)\right)
$$

Note that here the index $i$ runs from 1 to $2 n$, thereby allowing the possibility of admitting different weights for all the components of the vector $Z \equiv\{x, y\}$ where $z_{n+1} \equiv y_{i}, i=1,2,3, \ldots, n$.

\section{CONCLUSION}

In conclusion we want to stress the fact that the phenomenon of crime incidents and the associated criminal population is a dynamic process and can be very elegantly modeled and rigorously described using the modern theory of systems and control. But it appears from the literature that no such work has been done in the past. In this paper, for the first time, we have developed the mathematical framework of such a model. We have also shown its usefulness from the point of view of crime control and policy evaluation under any social and legal system. An actual application of this model using the Canadian correctional data base is currently being studied by the authors. 


\section{References}

[1] Stollmack, S. (1973), Predicting inmate population from arrest, court disposition, and recidivism rates, J. Res. Crime and Delinq., 10: 141-162.

[2] Blumstein, A., Cohen, J., Roth, J. and Visher, C.A. (1986), Criminal Careers and "Career Criminals", National Academy Press, Washington D.C., Vol. 1.

[3] Barnett, A. (1987), Prison population: A projection model, Opert. Res., 35(1): $18-34$.

[4] Schmidt, P. and Witte, A.D. (1988), Predicting Recidivism using Survival Models, Springer-Verlag, New York.

[5] Schmidt, P. and Witte, A.D. (1989), Predicting criminal recidivism using "Split Population Survival Time Models", J. Economet., 40: 141-159.

[6] Visher, C.A., Lattimore, P.K. and Linster, R.L. (1991), Predicting the recidivism of serious youthful offenders using survival models, Criminology, 29(3): 801-838.

[7] Lattimore, P.K. and Baker, J.R. (1992), The impact of recidivism and capacity on prison populations, $J$. of Quantitative Criminology, 8(2), 189-215.

[8] Lin, Bin-Shan, MacKenzie, D.L. and Gulledge, Jr., T.R. (1986), Using ARIMA models to predict prison populations, J. of Quantitative Criminology, 2(3): 251-264.

[9] Rahim, M.A., Recidivism, Proc. of the American Statistical Association, 1978, pp. 380-385.

[10] Rahim, M.A., On the Concept and Measurement of Recidivism - Current Practices and Suggested Improvements, Ministry of the Solicitor General of Canada, Vol. 41, 1984.

[11] Ahmed, N.U., Elements of Finite Dimensional Systems and Control Theory, Pitman Monographs on Pure and Applied Mathematics, Vol. 37, Longman Scientific and Technical and John Wiley, London, New York, (1988). 


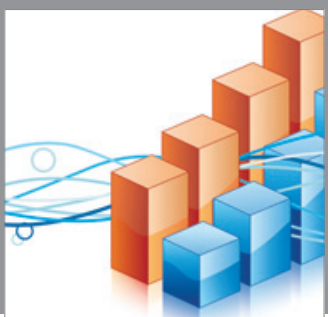

Advances in

Operations Research

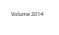

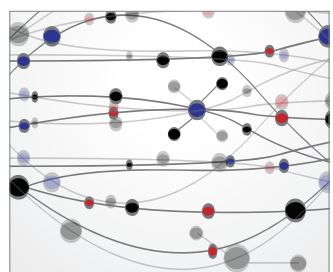

\section{The Scientific} World Journal
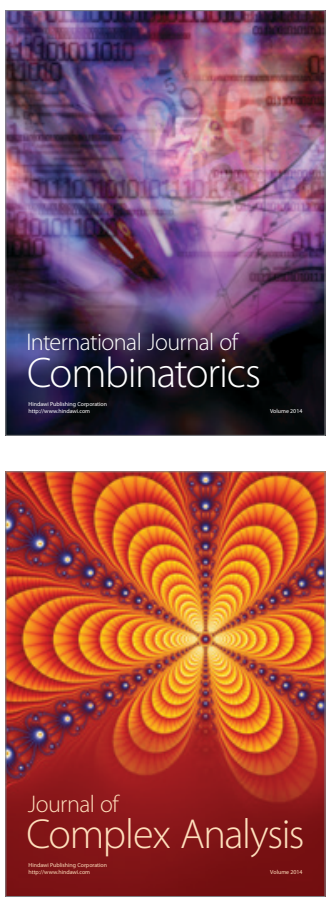

International Journal of

Mathematics and

Mathematical

Sciences
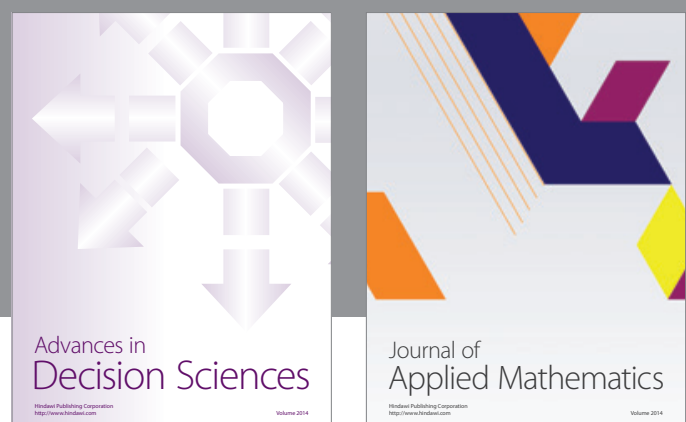

Journal of

Applied Mathematics
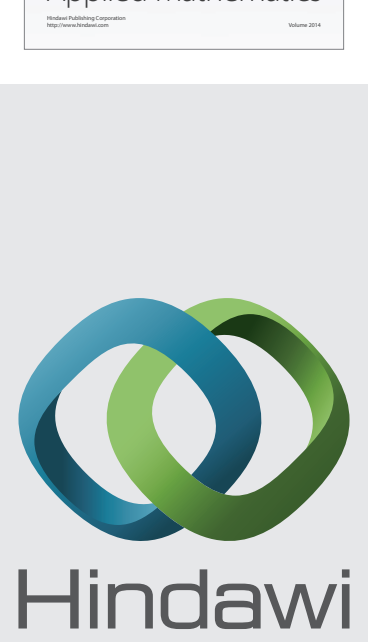

Submit your manuscripts at http://www.hindawi.com
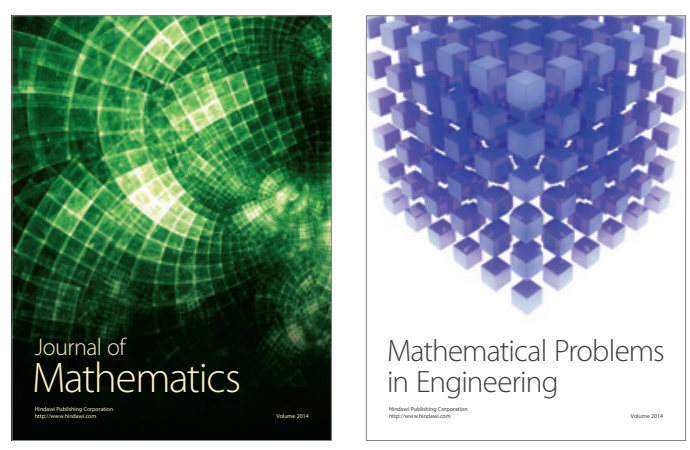

Mathematical Problems in Engineering
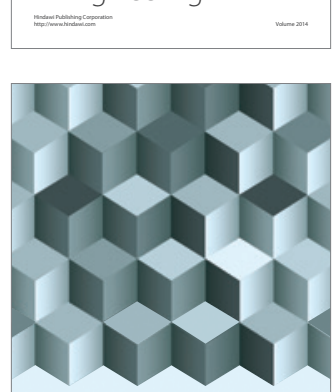

Journal of

Function Spaces
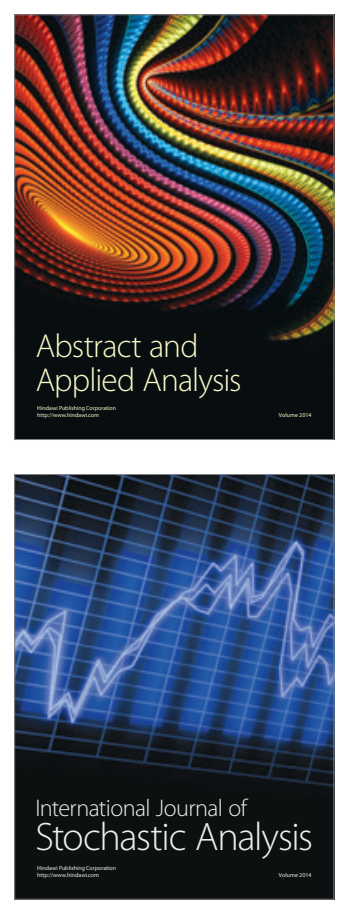

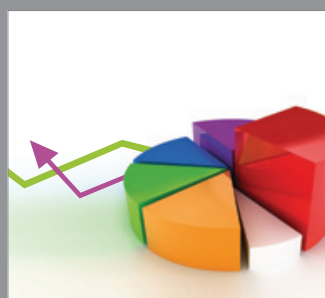

ournal of

Probability and Statistics

Promensencen
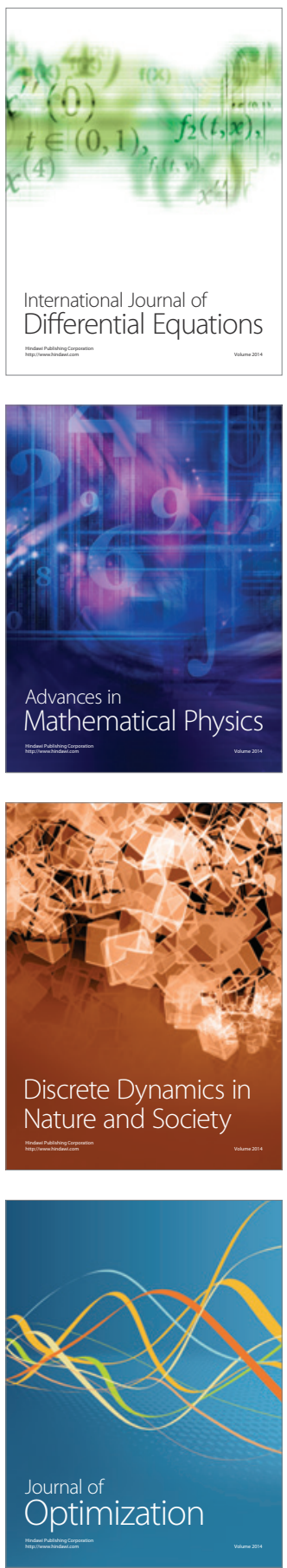